\section{ISG-003 BIOSIMILAR GROWTH HORMONE: DEFINED DAILY DOSE IN AN ITALIAN DISTRICT AFTER THE REGIONAL TENDER}

S Strobino*, M Lecis, E Viglione, G Ceravolo. ASL TO3, Hospital Pharmacy, Rivoli, Italy

10.1136/ejhpharm-2019-eahpconf.3

Background Somatotropic hormone is used in patients with growth disorders due to insufficient hormone uptake, Turner syndrome, chronic renal failure or Prader-Willi syndrome. In the Piedmont region, this drug is dispensed in the hospital pharmacy for patients who have a prescription charged to the National Health System. The biosimilar somatotropin was awarded in the regional tender, according to the economically most advantageous offer principle.

Purpose The objective of this work is the evaluation of the defined daily dose (DDD) of the somatotropic hormone of a Piedmont district, with regard to the DDD of the biosimilar somatotropin, in relation to the regional and Italian trends.

Material and methods The somatotropic hormone molecules' consumption in DDD of a Piedmont Hospital Pharmacy, of the region and of Italy between 2016 and 2017 were analysed.

Results In 2017 there was a DDD somatotropic hormone decrease compared to the previous year $(-8.6 \%)$, unlike the regional and national trend that did not see a significant difference in the two-year period considered. The somatotropina in 2016 recorded a DDD of 73.017, while in 2017 it had a DDD of 65.996 with a decrease of $-9.6 \%$. However, the molecule remains with $91.6 \%$ of the total DDD. The other molecules have a significantly lower DDD than the total ones of 2017: in fact in the second place, the octreotide is present only with $4.5 \%$.

Conclusion It is evident how high is the DDD of the somatotropin compared to the other same ATC[H1] class molecules' data. The prescribers shifted towards biosimilar thanks to the continuous work of information, updating and counselling of the hospital pharmacists. It is desirable to restore the Regional Register to improve the appropriateness in terms of doses and indications, and to evaluate constantly the epidemiological prescribing data.

\section{REFERENCES AND/OR ACKNOWLEDGEMENTS}

Biosimilar Position Paper AIFA.

No conflict of interest.

\section{ISG-004 ABSTRACT WITHDRAWN}


to two drugs, one patented (RPV) and one generic drug (FTC/TDF). Both were administered once-daily, providing the same therapeutic efficacy and treatment compliance but in a more cost-effective way.

Purpose To describe the procedure to implement this strategy and patient's acceptance of it.

Material and methods After several meetings between the Pharmacy (PD) and the Infectious Diseases Department (IDD) it was decided to make the change at the following patient's visit to the HS, either in the PD when the patient attended to pick up the medication or in the IDD in the patient's scheduled consultations.

Inclusion criteria: HIV patients treated with FTC/TDF/RPV up to June 2018 using e-prescribing records. Patients that did not contact our HS were excluded.

A retrospective review from July to October 2018 was conducted. Patients that would not accept the PD's change were referred to the IDD.

Collected data were: age, gender, treatment after the change and acceptance.

Results Out of 133 patients, seven were excluded. Mean age 47.6 years, $20 \%$ women. PD was responsible for $86 \%$ of the changes.

Out of 126 patients included, 16 (13\%) did not accept the change.

Of these 16 patients, five ended up accepting it (three after visiting the IDD and two on their second visit to the PD) and 11 declined to switch therapy for the following reasons: swallowing problems (one) (actual treatment: elvitegravir/cobicistat/ emtricitabine/tenofovir-alafenamide); adverse events (actual treatment: dolutegravir $\pm \mathrm{TDF} / \mathrm{FTC}$ (one); abacavir/lamivudine (two) or lamivudine (one); and six patients continued with FTC/TDF/RPV (four waiting for IDD next consultation and two due to medical decisions).

Conclusion By the time this abstract was written, the change was made in $115 / 126$ patients (91\%).

It is very important to highlight the efficient teamwork between the PD and the IDD in order to implement the new strategy in a short period of time.

Although initially $13 \%$ disagreed, finally only $9 \%$ of patients did not accept the proposed change.

On the other hand, this strategy has reduced the economic impact of HIV treatment in 51\% of patients.

\section{REFERENCES AND/OR ACKNOWLEDGEMENTS}

Infectious Diseases Department.

No conflict of interest.

\section{ISG-006 HEPATITIS C TREATMENT: COST AND EFFECTIVENESS}

A Brito*, A Fernandes, L Lourenço, S Domingos, A Soares, A Alcobia. Hospital Garcia de Orta, Pharmacy, Almada, Portugal

10.1136/ejhpharm-2019-eahpconf.6

Background The hepatitis C virus (HCV) infection is one of the major causes of chronic liver disease. Due to the HCV infection prevalence in European regions (1.5\%), increasing health expenditure has been made in order to eradicate HCV.

Purpose Assess the cost, effectiveness and safety of treated $\mathrm{HCV}$ patients with pegylated interferon-ribavirin (IFN-RIB) compared to new direct acting antivirals (DAA).
Material and methods Retrospective observational comparative study of two cohorts of patients including HCV patients who started and finished treatments with IFN-RIB in 2011 (except the above 12 months) and with DAAs in 2017. Exclusion criteria: deaths, no therapeutic adherence and transfer to another hospital. Data were extracted from electronic records.

Results In 2011, 49 patients $(87.8 \%$ male) with a mean age $44.4 \pm 7.1$ years were included: $12.2 \%$ were previously treated, $40.8 \%$ co-infected and genotypes 1 was predominant $(51.0 \%)$, and $73.5 \%$ were treated with IFN $\alpha 2 a-R I B$ and the remaining with IFN $\alpha 2$ b-RIB.

In 2017, 185 patients $(75.0 \%$ male) were included, with a mean age $52.2 \pm 9.9$ years, $28.1 \%$ co-infected. Genotype 1 $(64.9 \%)$ was the most common: $79.3 \%$ patients had severe or moderate fibrosis (FD $\geq 2$ ). Only $11.4 \%$ were previously treated (four with DAA). Treatments were: 71.9\% Ledipasvir/ Sofosbuvir; 7.6\% Sofosbuvir/Velpatasvir; 7.0\% Sofosbuvir; 7.0\% Elbasvir/Grazoprevir; 3.2\% Ombistasvir/Paritaprevir/Ritonavir+Dasabuvir; $2.2 \%$ Daclatasvir +Sofosbuvir; $0.5 \%$ Sofosbuvir +Ombistasvir/Paritaprevir/Ritonavir+Dasabuvir; and 0.5\% Ombistavir/Paritaprevir/Ritonavir.

Comparing treatments of two cohort patients (IFN-RIB/ DAA): in 2011 average treatment length was 8.1 months/ patient much longer than 2017 (3.5 months/patient) and 12 weeks' length in $70.4 \%$. In 2011, drug discontinuation occurred in $36.7 \%$ treatments because the patients had serious adverse reactions (AR) or were non-responders. In 2017, DAA had fewer and lower severity AR (100\% compliance). According to guidelines' alterations, eight patients had shortened their initial duration of treatment. Treatments with IFN-RIB (€ 4287.7/patient) were less expensive than DAA (€ 14867.1/patient), representing an increase of $€ 1309.2 \%$ annually. Although the success rate was significantly higher with DAA $(96.8 \%)$ than with IFN-RIB (53.1\%), 23/49patients, in 2011, were posteriorly treated with DAA. The incremental cost-effectiveness ratio (DAA/ IFN-RBV) was $€ 238.1$ patient successfully treated. Costs are higher, but, in 2018, the costs of treatment/patient are half that of 2017.

Conclusion DAA treatments have higher effectiveness against HCV infection(>95\%) and treatments are shorter, more effective and safer than older therapies, despite higher costs.

\section{REFERENCE AND/OR ACKNOWLEDGEMENTS}

WHO Guidelines for the screening, care and treatment of persons with chronic HCV infection 2016.

No conflict of interest.

\section{ISG-007 PEMETREXED'S LESSON}

AM Soares* , A Alcobia. Hospital Garcia de Orta, Pharmacy Department, Almada, Portugal

10.1136/ejhpharm-2019-eahpconf.7

Background In May 2008, the European Medicines Agency (EMA) granted authorisation to Pemetrexed as a first-line treatment for locally advanced or metastatic non-small cell lung cancer (NSCLC), other than predominantly squamous cell (non-SC) histology patients. A phase III trial compared Pemetrexed with Gemcitabine, both in association with cisplatin, and found a similar overall survival between both groups (10.3 months). The EMA authorisation was only based in a 